\title{
Radio frequency induced and neoclassical asymmetries and their effects on turbulent impurity transport in a tokamak
}

\author{
István Pusztai ${ }^{1,2, *}$, Matt Landreman ${ }^{3}$, Albert Mollén ${ }^{1}$, Yevgen O. Kazakov ${ }^{4}$, and Tünde Fülöp ${ }^{1}$ \\ ${ }^{1}$ Applied Physics, Chalmers University of Technology and Euratom-VR Association, SE-41296 Göteborg, \\ Sweden \\ ${ }^{2}$ Plasma Science and Fusion Center, Massachusetts Institute of Technology, Cambridge, MA 02139, USA \\ ${ }^{3}$ University of Maryland, College Park, MD 20742, USA \\ ${ }^{4}$ Laboratory for Plasma Physics, ERM/KMS, Association 'EURATOM-Belgian State', TEC Partner, BE-1000 \\ Brussels, Belgium
}

Received XXXX, revised XXXX, accepted XXXX

Published online XXXX

Key words tokamak, impurity transport, poloidal asymmetries, gyrokinetic, neoclassical, peaking factor

Poloidal asymmetries in the impurity density can be generated by radio frequency heating in the core and by neoclassical effects in the edge of tokamak plasmas. In a pedestal case study, using global neoclassical simulations we find that finite orbit width effects can generate significant poloidal variation in the electrostatic potential, which varies on a small radial scale. Gyrokinetic modeling shows that these poloidal asymmetries can be strong enough to significantly modify turbulent impurity peaking. In the pedestal the $E \times B$ drift in the radial electric field can give a larger contribution to the poloidal motion of impurities than that of their parallel streaming. Under such circumstances we find that up-down asymmetries can also affect impurity peaking.

Copyright line will be provided by the publisher

\section{Introduction}

Close to the magnetic axis in tokamaks, where the radial gradients of plasma parameters are too weak to destabilize microinstabilities [1], the transport of highly charged impurities is dominated by neoclassical processes [2]. For typical density and temperature profiles this leads to an accumulation of impurities in the deep core. The accumulation not only reduces the fusion performance by diluting the plasma, but can lead to radiative cooling and trigger magnetohydrodynamic activity [3]. Tungsten excursions and radiative events are observed in recent experiments operating with tungsten plasma facing components [4]. The possibility of accumulation of highly charged impurities in the core of reactor relevant tokamak plasmas, such as in ITER, is a serious issue [5]. Therefore significant effort has been dedicated to find scenarios or active methods to reduce core impurity accumulation.

Auxiliary radio frequency (RF) heating in the ion- and electron cyclotron frequency ranges has been demonstrated as a possibility of reducing impurity accumulation [6-8]. However the physical processes responsible for changing impurity transport are not fully understood. In the region very close to the axis where turbulence is not the dominant transport channel, changes in neoclassical transport or magnetohydrodynamic activity may be candidates [4]. A bit further out from the center, changes in the turbulence properties can also play an important role. Recent studies pointed towards a possible role of poloidal asymmetries which can be generated in RF heated plasmas [9-13]. In plasmas lacking strong toroidal rotation the densities of the different species are usually assumed to be approximately flux functions. However a temperature anisotropy of the heated species can lead to a poloidal variation of the density of that species, which generates a poloidally varying electrostatic field [14]. This field may be weak to leave the dynamics of the main species practically unaffected, but the response of a highly charged impurity species to these fields can be significant. The distribution of impurities on the flux surface can develop significant poloidal asymmetries, and their $\mathbf{E} \times \mathbf{B}$ drift in the poloidally varying electrostatic field can compete with the magnetic drifts [11], which has important consequences on the turbulent impurity transport.

\footnotetext{
* Corresponding author E-mail: pusztai@chalmers.se, Phone: +1 617253 5799, Fax: +1 6172530238
} 
The local value of the steady state radial logarithmic impurity density gradient (referred to as the impurity peaking factor), is determined by the local turbulent transport. On the one hand, impurity transport in the plasma edge provides a boundary condition for the impurity density profile, thus affecting impurity accumulation in the core. On the other hand, a relatively high impurity concentration may be necessary in the edge for reactor relevant devices to reduce localized heat loads on plasma facing components through radiation. It is therefore of interest to know the impurity transport in the edge. It would be an unreasonably large undertaking to give a complete description of the edge impurity transport in one paper given the complex nature of the problem. Instead, we will consider certain aspects of the problem related to poloidal asymmetries.

There are experimental observations of strong poloidal in-out asymmetries in the impurity density in the edge plasma [15-17], especially in the strong gradient regions of edge transport barriers (henceforth, we refer to the inboard/outboard impurity accumulation as in-out/out-in density asymmetry). In the same time, neoclassical theory also predicts poloidal impurity density asymmetries [18,-20]. It has to be noted that the poloidal asymmetries experimentally observed in the pedestal can be several times larger [16] than what the theory described in Refs. [18-20] predicts. This may partly be due to the simplified magnetic geometries considered, but more likely the reason is that kinetic effects associated with finite ion orbit width [21] in the pedestal are not accounted for.

In the present paper we first give an overview of research done on turbulent impurity transport in the presence of poloidal asymmetries generated by ion cyclotron resonance heating (ICRH). Then we consider the effect of edge asymmetries due to neoclassical effects. We use a global numerical tool for neoclassical calculations in the pedestal PERFECT (Pedestal \& Edge Radially global Fokker-Planck Evaluation of Collisional Transport) [22], and a combination of gyrokinetic simulations and semi-analytical modeling to study the turbulent transport in the presence of poloidal asymmetries and strong radial electric fields.

The subsequent sections are organized as follows. In Sec. 2 different mechanisms leading to poloidal impurity asymmetries in the core and edge are reviewed. Then, the gyrokinetic modeling of turbulent impurity transport in the presence of asymmetries is described in Sec. 3 Finally, simulation results are presented and interpreted in Sec.4 before we draw our conclusions in Sec. 5.

\section{Asymmetries in the core and in the edge}

In numerical ICRH simulations the non-Maxwellian part of the distribution function of the heated ion species (denoted by subscript $m$, as minority) is often found to exhibit velocity space structures that can be well approximated by the ansatz [23] $f_{m} \propto \exp \left[-\mu B_{c} / T_{\perp}-\left|\mathcal{K}-\mu B_{c}\right| / T_{\|}\right]$, where $\mathcal{K}=m_{m} v^{2} / 2, \mu=m_{m} v_{\perp}^{2} /(2 B)$, and $T_{\perp}$ and $T_{\|}$are radially varying parameters representing the perpendicular and parallel temperatures, respectively (note that the temperature anisotropy $\alpha_{T}=T_{\perp} / T_{\|}>1$ due to the ICRH heating). Furthermore, the magnetic field strength $B$ is equal to $B_{c}$ where the ICRH absorption is the largest on the flux surface. The density moment of this distribution function yields a poloidally varying minority density with a maximum close to the poloidal angle $\theta_{0}$ where $B\left(r, \theta_{0}\right)=B_{c}$, which reflects that the heated particles tend to get trapped due to the heating with their banana tips close to the ICRH resonance location. In particular, when the $B=B_{c}$ surface is tangential to the studied flux surface from the low magnetic field side (this is the situation considered unless stated otherwise), the density of the heated minority species has a maximum at the outboard mid-plane $\left(\theta_{0}=0\right)$. The corresponding poloidally varying electrostatic potential $\phi_{E}$, that sets up to sustain quasineutrality, has a minimum on the inboard side. Consequently the impurities accumulate in that region.

In a large aspect ratio circular cross section plasma the poloidal variation of the impurity density is approximately sinusoidal $n_{z}(r, \theta)=n_{z 0}(r) \exp \left[-Z e \phi_{E}(r, \theta) / T_{z}(r)\right] \approx n_{z 0}(r) \exp [K \cos (\theta-\bar{\theta})]$, where $n_{z 0}$ (sometimes referred to as the pseudo density) is a flux function, $Z$ is the impurity charge number, and $T_{a}$ denotes the temperature of species $a$. Furthermore $\theta=\bar{\theta}$ corresponds to the poloidal location of the maximum of the impurity density. The asymmetry strength $K$ can be approximated as $K=Z k\left(n_{m 0} / n_{e}\right) /\left[\left(n_{i} / n_{e}\right)+\left(T_{z} / T_{e}\right)+\right.$ $\left.\left(n_{z 0} Z^{2} / n_{e}\right)\right]$, with $k=\left[\epsilon b_{c}\left(\alpha_{T}-1\right)\right] /\left[b_{c}+\alpha_{T}\left(1-b_{c}\right)\right]$ [24]. Here, $n_{m 0}$ is the flux surface average of the minority density, $\epsilon=r / R_{0}$ with $R_{0}$ the major radius of the centroid of the flux surface, $b_{c}=B_{c} / B_{0}$ with $B_{0}=B\left(R_{0}\right)$. We assume $T_{z}=T_{i}$ and that the poloidal variations of the main ion and electron densities $-n_{i}$ and $n_{e}$, respectively - are negligible.

In the edge, where the plasma parameter profiles may be sharp due to transport barriers there can be various effects which give rise to poloidal asymmetries in the impurity density. In the parallel impurity momentum 
equation normally the electric field and the pressure gradient terms balance, $T_{i} \nabla_{\|} n_{z}=-e_{z} n_{z} \nabla_{\|} \tilde{\phi}$, leading to a Boltzmann response of the impurities (assuming $T_{z}=T_{i}$ to be a flux function). This means that even if the impurities are not too collisional, there is a possibility to develop poloidal impurity density asymmetries, since parallel electric fields can be present due to poloidal asymmetries in the main ion density. These asymmetries naturally arise when the main ions are in the plateau or Pfirsch-Schlüter regime of collisionality even in the presence of weaker gradients. The form of this density variation is $\propto \sin \theta[18]$. When the gradients and the collisionality are sufficiently high, so that the impurity-ion friction becomes comparable to the parallel pressure gradient term in the parallel momentum balance equation, that can also lead to poloidal impurity density variations. In this case, if the main ions are either in the banana or in the Pfirsch-Schlüter regimes, the impurities are pushed to the inboard side [19, 20], whereas in the plateau regime, there can be in-out or out-in impurity density asymmetry depending on the ratio of the density and temperature scale lengths, the strength of the impurity ion friction, and the impurity concentration [18]. Finite Mach number effects can also modify the poloidal density variation and the neoclassical transport of impurities [19].

Neoclassical calculations and simulation codes normally assume that the orbit width of particles (which, in general, is comparable to their poloidal Larmor radius $\rho_{\theta a}=\left(B / B_{\theta}\right) \rho_{a}$, where $B_{\theta}$ is the poloidal magnetic field) is much smaller than the radial scale length of plasma parameters. In this case a local analysis (i.e. considering a single flux surface) is sufficient. In very sharp pedestals this assumption may break down, first for the main ions (and low charge number impurities). If the ion temperature scale length $L_{T i}=-\left[\partial_{r}\left(\ln T_{i}\right)\right]^{-1}$ becomes comparable with $\rho_{\theta i}$, then the distribution function may not remain close to a Maxwellian anymore, which requires a global, nonlinear description. However, if only the electron density and temperature scale lengths $\left(L_{n e}=-\left[\partial_{r}\left(\ln n_{e}\right)\right]^{-1}\right.$ and $\left.L_{T e}=-\left[\partial_{r}\left(\ln T_{e}\right)\right]^{-1}\right)$ are comparable to $\rho_{\theta i}$, although a global description is still necessary, the distribution function can be expanded about a Maxwellian, and the linear drift-kinetic equation may be employed [22]. This limit, apart from being convenient for theoretical studies, has physical relevance as well; the ion temperature scale length often appears to be much larger than the density scale length in pedestals of DIII-D [25,26]. Furthermore, in very large aspect ratio $\sqrt{\epsilon} \ll 1$, the scale separation between the ion orbit width $\sim \rho_{\theta i} \sqrt{\epsilon}$ and $\rho_{\theta i}$ allows for simplifications making an analytical treatment possible [21,27,28]. In pedestals with realistic aspect ratio further poloidal asymmetries in the main ion flow [22] and density can arise. To calculate these asymmetries one needs to resort to numerical simulations.

\section{Gyrokinetic modeling}

We consider the peaking of a highly charged trace impurity species sustained by the linear flux of a single unstable mode. Specifically, to determine the impurity peaking factor in steady state we require the flux surface average of the radial linear impurity flux to vanish $\Gamma_{z} \equiv-\operatorname{Im}\left\langle\left(k_{y} / B\right) \int d^{3} v J_{0}\left(z_{z}\right) g_{z} \phi^{*}\right\rangle$. Here $\langle\cdot\rangle$ denotes the flux surface average, $k_{y}=n q / r$ is the binormal wave number with $n$ being the toroidal mode number and $q$ the safety factor, and $\phi^{*}$ is the complex conjugate of the perturbed electrostatic potential. The impurity finite Larmor radius (FLR) parameter $z_{z}$, appearing in the argument of the Bessel function of the first kind $J_{0}$, is defined as $z_{z}=k_{\perp} v_{\perp} / \Omega_{z}$ with $k_{\perp}=k_{y}\left(1+s^{2} \theta^{2}\right)^{1 / 2}$ the perpendicular wave number, $s$ the magnetic shear and $\Omega_{a}=e_{a} B / m_{a}$ the cyclotron frequency of species $a$, where $e_{a}$ and $m_{a}$ are the species charge and mass.

We formulate the problem in the laboratory frame, which is convenient for a pedestal with subsonic ion rotation. We consider the situation when the large diamagnetic and $\mathbf{E} \times \mathbf{B}$ rotation speeds nearly cancel for the main ions, and the impurities are collisionally coupled to them thereby having a similar flow speed and temperature. The non-adiabatic part of the perturbed impurity distribution function $g_{z}$ is governed by the linear gyrokinetic (GK) equation. Considering a circular cross section large aspect ratio plasma the GK equation reads

$$
\left(v_{\|}+u\right) \mathbf{b} \cdot \nabla \theta \partial_{\theta} g_{z}-i\left(\omega-\omega_{D z}-\omega_{E}-\tilde{\omega}_{E r}\right) g_{z}-C_{z}^{(l)}\left[g_{z}\right]=-i\left(e_{z} \phi / T_{z}\right) f_{z 0}\left(\omega-\omega_{* z}^{T}-\tilde{\omega}_{E r}\right) J_{0}\left(z_{z}\right),
$$

where $\mathbf{b} \cdot \nabla \theta=1 /\left(q R_{0}\right)$, with $\mathbf{b}=\mathbf{B} / B$ the unit vector along the magnetic field $\mathbf{B}$, and $C_{z}^{(l)}$ is the linearized impurity collision operator. The parallel gradient is to be taken at fixed total unperturbed energy $\mathcal{E}=m_{z} v^{2} / 2+$ $e_{z} \phi_{E}$ and magnetic moment $\mu=m_{z} v_{\perp}^{2} /(2 B)$. We split $\phi_{E}=\Phi(r)+\tilde{\phi}(r, \theta)$, where $\langle\tilde{\phi}\rangle=0$. The contribution of the $\mathbf{E} \times \mathbf{B}$ drift to the poloidal motion is represented by $u \approx B_{\theta}^{-1} \partial_{r} \Phi$, the Doppler shifted frequency is $\omega=\underline{\omega}-\omega_{E r}$ with $\underline{\omega}$ the laboratory frame frequency and $\omega_{E r}=\left(k_{y} / B\right) \partial_{r} \Phi$, while $\tilde{\omega}_{E r}=\left(k_{y} / B\right) \partial_{r} \tilde{\phi}$. The radial drift due to the poloidal electric field is $\omega_{E}=-k_{y} s \theta\left(\partial_{\theta} \tilde{\phi}\right) /(B r)$. The magnetic and diamagnetic 
drift frequencies are defined as $\omega_{D z}=-\left(k_{y} / \Omega_{z} R\right)\left(v_{\|}^{2}+v_{\perp}^{2} / 2\right)(\cos \theta+s \theta \sin \theta)$ and $\omega_{* z}^{T}=\omega_{a z}\left\{\left(a / L_{n z}\right)+\right.$ $\left.\left[m_{z} v^{2} /\left(2 T_{z}\right)-3 / 2\right]\left(a / L_{T z}\right)\right\}$, where $\omega_{a z}=-k_{y} T_{z} /\left(e_{z} B a\right)$ with $a$ the plasma minor radius, and $1 / L_{n z}=$ $-\partial_{r}\left(\ln n_{z}\right)$ defines the impurity density-, and $1 / L_{T z}=-\partial_{r}\left(\ln T_{z}\right)$ the impurity temperature scale lengths; note that $1 / L_{n z}$ is not a flux function. The lowest order distribution function is a Maxwellian $f_{z 0}(r, \mathcal{E})=$ $n_{z 0}\left(m_{z} / 2 \pi T_{z}\right)^{3 / 2} \exp \left(-\mathcal{E} / T_{z}\right)=n_{z}\left(m_{z} / 2 \pi T_{z}\right)^{3 / 2} \exp \left(-m_{z} v^{2} /\left(2 T_{z}\right)\right)$, where $n_{z 0}=n_{z} \exp \left(e_{z} \phi_{E} / T_{z}\right)$ is a flux function. In the right hand side of the gyrokinetic equation $\omega-\omega_{* z}^{T}-\tilde{\omega}_{E r}$ stems from $\underline{\omega}-\tilde{\omega}_{* z}^{T}$, where $\tilde{\omega}_{* z}^{T}=-\omega_{a z} a\left[\partial_{r}\left(\ln n_{z 0}\right)+\left(\mathcal{E} / T_{z}-3 / 2\right) \partial_{r}\left(\ln T_{z}\right)\right]$.

We solve (11) perturbatively keeping $\omega$ and the impurity-impurity collisions in $0^{\text {th }}$ order, the $v_{\|}$and $u$ terms in the $1^{\text {st }}$ order, and the $\omega_{D z}, \omega_{E}, \tilde{\omega}_{E r}, \omega_{* z}^{T}$ and $J_{0}\left(z_{z}\right)-1$ terms to $2^{\text {nd }}$ order. This reduces to an expansion in $Z^{-1 / 2}$ in the plasma core, consistent with $e_{z} \partial_{\theta}(\tilde{\phi}) / T_{z} \sim 1$ (see [13] for details), but in the pedestal where radial scale lengths of $n_{Z}$ and $e_{z} \tilde{\phi} / T_{z}$ are comparable to $\rho_{\theta i}$ it is an ad-hoc ordering to be justified a posteriori by numerical results. The largest term in the impurity-ion collision operator is a friction term of the form $\propto$ $\nabla_{v} f_{z}(\mathbf{v}) \cdot \int d^{3} v^{\prime} f_{i}\left(\mathbf{v}^{\prime}\right) \mathbf{v}^{\prime} / v^{\prime 3}$ that includes the perturbed ion distribution $f_{i}$. We assume that this term acts to make the impurity flow speed sufficiently close to the main ion flow speed so that $C_{z i}^{(l)}$ only needs to be included in the perturbative scheme at $2^{\text {nd }}$ order. When ordering $C_{z i}^{(l)}$ this way and using the conservation properties of $C_{z z}^{(l)}$, collisions do not affect the turbulent impurity flux to the accuracy of the model.

Since $n_{z}$ varies over the flux surface we define an effective impurity peaking factor as $a / L_{n z}^{0}=a / L_{n z}^{*}$ when $\Gamma_{z}=0$, where $a / L_{n z}^{*}=\left\langle a / L_{n z}\right\rangle_{\phi}$ with the weighted flux surface average $\langle\ldots\rangle_{\phi}=\left\langle\ldots \mathcal{N}|\phi|^{2}\right\rangle /\left\langle\mathcal{N}|\phi|^{2}\right\rangle$ and $\mathcal{N}(\theta)=\exp \left(-e_{z} \tilde{\phi} / T_{z}\right)$. Assuming $Z e \tilde{\phi} / T_{z}=-K \cos (\theta-\bar{\theta})$ and neglecting $\mathcal{O}(\epsilon)$ corrections we find

$$
\begin{aligned}
\frac{a}{L_{n z}^{0}} & =2 \frac{a}{R_{0}}\langle\mathcal{D}\rangle_{\phi}+\frac{a}{r} s K\langle\theta \sin (\theta-\bar{\theta})\rangle_{\phi} \\
& -\left(\frac{m_{i}}{m_{z}}+2 U_{i}^{2}\right) \frac{2 Z a v_{i}}{q^{2} R_{0}^{2} k_{y} \rho_{i}} \frac{\omega_{r}}{|\omega|^{2}}\left\langle\frac{\left|\partial_{\theta} \phi\right|^{2}}{|\phi|^{2}}\right\rangle_{\phi}+\frac{2 U_{i} Z a}{q R_{0} k_{y} \rho_{i} \operatorname{Im}\left[\omega^{-1}\right]}\left\langle\operatorname{Im}\left[\frac{i}{\omega} \frac{\partial_{\theta} \phi}{\phi}\right]\right\rangle_{\phi},
\end{aligned}
$$

where $\mathcal{D}=\cos \theta+s \theta \sin \theta$, the real part of $\omega$ is $\omega_{r}, U_{i}=u / v_{i}$, and $\rho_{i}=v_{i} m_{i} / e B_{0}$ with $v_{i}^{2}=2 T_{i} / m_{i}$. The first and second terms of (2) represent contributions from the magnetic drifts and the $\mathbf{E} \times \mathbf{B}$ drift in the poloidal electric field, respectively. The terms in the second line of (2) are contributions from the poloidal motion of the particle due to parallel streaming and the $\mathbf{E} \times \mathbf{B}$ drift in the radial electric field. The $U_{i}^{2}$ and $U_{i}$ terms can be important in the pedestal where $U_{i}$ can reach $\mathcal{O}(1)$ values. Note that if $\phi$ is symmetric around $\theta=0$ the last term of Eq. (2) is non-zero only if $\tilde{\phi}$ is not even in $\theta$ (i.e. there is a finite up-down asymmetry). Outside the pedestal $\left(U_{i} \ll 1\right)$ up-down asymmetric potentials have negligibly small effect on the turbulent impurity transport as demonstrated in [12].

\section{Simulation results and interpretation}

In this section we demonstrate the importance of neoclassical asymmetries on turbulent impurity transport in an edge transport barrier through numerical modeling.

The modeling is done in two steps. To calculate the neoclassical poloidally varying electrostatic potential $\tilde{\phi}$, first we use the code PERFECT [22], which is a radially global, $\delta f$, continuum neoclassical solver. PERFECT allows the electron density and temperature scale lengths to be comparably small to the poloidal ion Larmor radius, $\rho_{\theta i}$, while requiring the ion temperature to vary slowly on that scale. The potential $\tilde{\phi}$ is then used as an input to the modeling of the turbulent impurity transport.

We consider plasma parameter profiles that resemble experimental profiles in H-mode pedestals of the DIII-D tokamak, although they do not represent any specific experiment. The neoclassical simulations aim to show that it is plausible for finite-orbit-width neoclassical physics to generate in-out and/or out-in asymmetries; indeed these asymmetries are observed for a wide range of input profiles in PERFECT. Figure 1 shows the variation of the most important plasma parameters across the simulation domain in the PERFECT simulation. The ion and electron temperatures, which are assumed to be equal, vary slowly over the simulation domain, as shown by the dashed curve in Fig. 11. However, the electron density has a significant drop around $\psi_{N}^{1 / 2}=0.99$ (solid curve). Accordingly, $a / L_{n}\left(\equiv a / L_{n e} \approx a / L_{n i}\right)$ greatly exceeds $a / L_{T}\left(\equiv a / L_{T i}=a / L_{T e}\right)$ in this region, as 

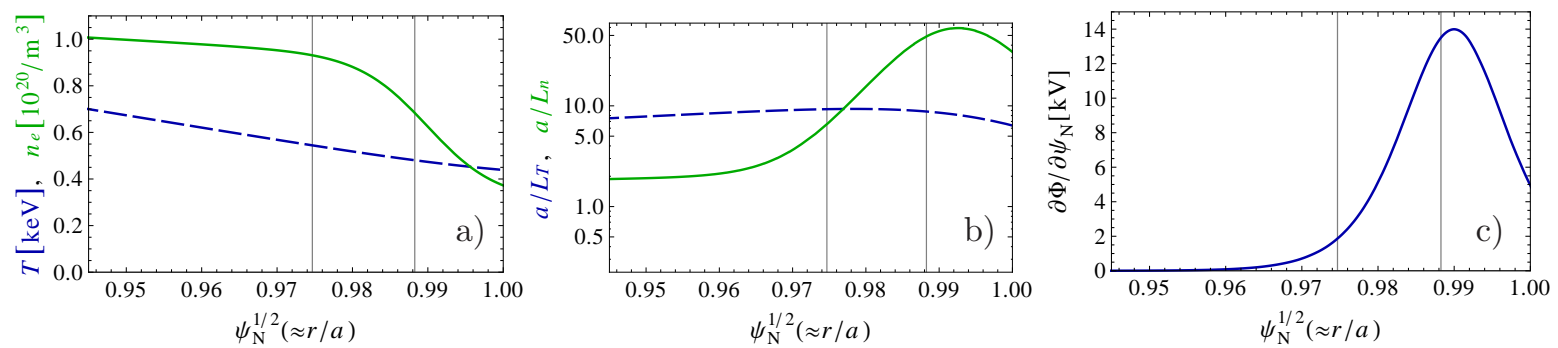

Fig. 1 Variation of plasma parameters across the simulation domain, used as inputs for the PERFECT simulation. The following quantities are depicted: a) temperature in $\mathrm{keV}$ [dashed curve], electron density in $10^{20} \mathrm{~m}^{-3}$ [solid curve], b) $a / L_{T}$ [dashed], $a / L_{n}$ [solid], c) radial variation of the electrostatic potential in $\mathrm{kV}$.

shown in Fig.11b. The maximum value of $\rho_{\theta i} / L_{n}$ and $\rho_{\theta i} / L_{T}$ in the domain are 0.92 and 0.16 , respectively. The impurities are assumed to be trace in the sense that they do not affect quasineutrality, hence the electron and ion density profiles are approximately the same. In particular, the PERFECT simulation was performed for a pure plasma. The simulation uses a Miller model equilibrium and the variation of the magnetic geometry parameters across the domain is neglected. The aspect ratio is $R / a=3.17$, the radial variation of the Shafranov shift is $\partial_{r} R_{0}=-0.354$, the Miller parameters [29] for elongation are $\kappa=1.66$ and $s_{\kappa} \equiv\left(r \partial_{r} \kappa\right) / \kappa=0.7$, and those for the triangularity are $\delta=0.416$ and $s_{\delta} \equiv\left(r \partial_{r} \delta\right) /\left(1-\delta^{2}\right)^{1 / 2}=1.37$, finally, the safety factor is $q=4$, and the magnetic shear is unimportant for the neoclassical simulation. The radial variation of the potential $\Phi$ corresponding to the radial electric field is not calculated in PERFECT but rather it needs to be provided as an input for the calculation; see Fig. 11.

The converged resolution parameters of the simulation are the following. The number of poloidal modes is $N_{\theta}=25$, the number of Legendre polynomials used in the Rosenbluth potentials is $N_{L}=4$, the number of grid points in $v, \xi=v_{\|} / v$ and normalized poloidal flux $\psi_{N}$ (the radial coordinate) are $N_{v}=6, N_{\xi}=13$ and $N_{\psi}=55$, respectively. The domain size in speed is $v_{\max }=5 v_{i}$. The simulation considered the dynamics of a single ion species, deuterium.

Out of the several outputs of the PERFECT simulation we only need the poloidally varying electrostatic potential $\tilde{\phi}$ that is generated by the perturbed ion density. This quantity is shown in Fig. $2 \mathrm{k}$ as a function of $\sqrt{\psi_{N}}$ and $\theta$. The red (blue) tones correspond to positive (negative) values of the potential. The most conspicuous feature of $\tilde{\phi}$ is that the poloidal location of the minimum of the potential can vary rapidly radially. On the inner boundary of the simulation domain there is a moderate up-down asymmetry, which transits to a stronger in-out asymmetry. Then around the location of the pedestal top the polarity flips from in-out to an out-in asymmetry which, just inside the last closed flux surface (LCFS) flips back to an in-out asymmetry.

Since up-down asymmetries in $\tilde{\phi}(\theta)$ are shown to have only a very weak effect on turbulent impurity transport if $U_{i} \ll 1$ we concentrate on areas with in-out or out-in asymmetries. It might be worth emphasizing that conventional neoclassical theory based on the assumption of $L_{T} \sim L_{n} \gg \rho_{\theta i}$ can provide only up-down potential asymmetry (as long as the flux surface is not strongly up-down asymmetric). Accordingly, an advanced theory or simulation code is necessary to compute $\tilde{\phi}(\theta)$. We choose two radial locations for further studies from the confined region $\left(\psi_{N}<1\right)$ where the asymmetries are the strongest. These locations, $\psi_{N}^{1 / 2}=0.975$ and $\psi_{N}^{1 / 2}=$ 0.988, are marked with vertical bars in Fig. 2 a, and in Fig. 1 as well. In the following we will calculate the effective impurity peaking factors at these two locations using the model presented in Section 3 As an impurity we consider fully ionized nickel $(Z=28)$. For this charge number, the poloidal variation of $e_{z} \tilde{\phi} / T_{z}$ is shown in Fig. $2 \mathrm{~b}$ for the two radial locations. We approximate the potential variation with the sinusoidal model given above Eq. (2); the corresponding fitted parameters for $\psi_{N}^{1 / 2}=0.975$ are $K=0.610$ and $\bar{\theta} / \pi=0.885$, and for $\psi_{N}^{1 / 2}=0.988$ are $K=0.676$ and $\bar{\theta} / \pi=0.084$ (the fits are represented by the dashed lines in Fig. 2 2 b).

Toroidal rotation and rotation shear can modify heavy impurity transport significantly, as demonstrated in Ref. [30] and references therein. Assuming the parallel ion and impurity flow speeds to be comparable, the 

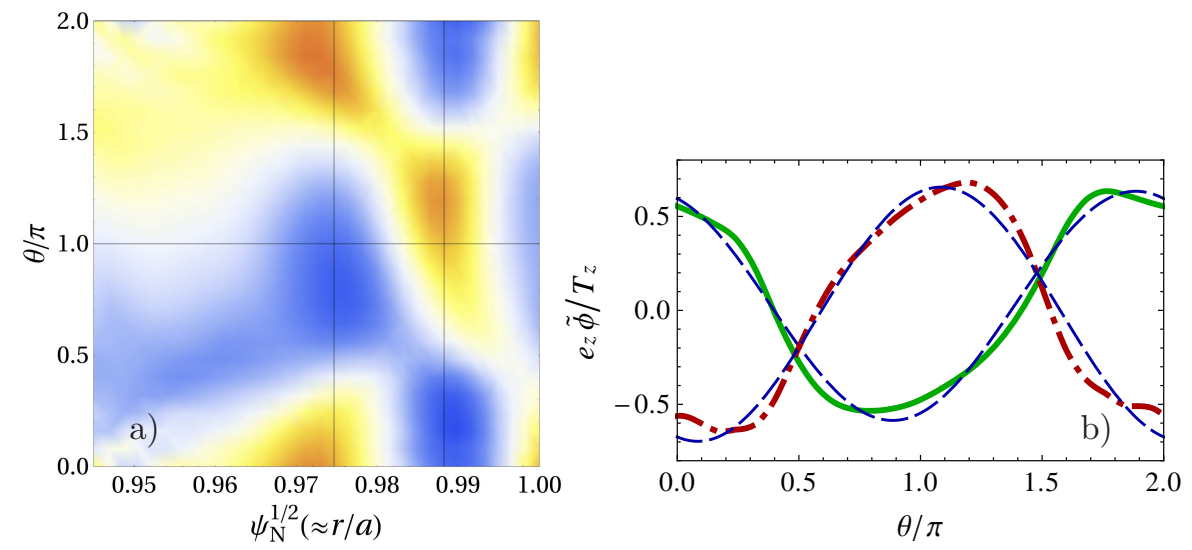

Fig. 2 a) Perturbed electrostatic potential, $\tilde{\phi}$, as a function of radius and poloidal angle (output of the PERFECT simulation): the largest positive (red) value is $15.2 \mathrm{~V}$, the highest negative (blue) value is $-11.5 \mathrm{~V}$. The vertical bars mark the two studied radial locations, $\psi_{N}^{1 / 2}=0.975$ and $\psi_{N}^{1 / 2}=0.988$. b) The poloidal variation of $e_{z} \tilde{\phi} / T_{z}$ at $\psi_{N}^{1 / 2}=0.975$ (solid line) and at $\psi_{N}^{1 / 2}=0.988$ (dash-dotted line), together with corresponding sinusoidal fits (thin dashed lines).

square of the impurity Mach number is $M_{z}^{2} \approx 0.16$ on flux surface average at these locations. This may be nonnegligible, but it is expected to have a smaller effect on the impurity peaking factor than that of the poloidally varying electrostatic potential. For simplicity the radial coordinate $r / a$ is taken to be equal to $\psi_{N}^{1 / 2}$.

The local plasma parameters used in the simulations at the two radial locations are the following. At $r / a=$ $0.975, T_{i} / T_{e}=1, a / L_{n e}=a / L_{n i}=6.59, a / L_{T e}=a / L_{T i}=9.27$, and the electron-ion collision frequency is $\nu_{e i}\left[c_{s} / a\right]=1.72$, where $c_{s}=\left(T_{e} / m_{i}\right)^{1 / 2}$ is the ion sound speed. At $r / a=0.988, T_{i} / T_{e}=1, a / L_{n e}=$ $a / L_{n i}=48.7, a / L_{T e}=a / L_{T i}=8.78$, and $\nu_{e i}\left[c_{s} / a\right]=1.62$. The same magnetic geometry parameters are used as in the neoclassical simulation, and additionally we set the magnetic shear $s=5$ in both radial locations.

We use local, electrostatic gyrokinetic simulations with the GYRO code [31] to calculate the mode frequency $\omega=\omega_{r}+i \gamma$ and the eigenfunction $\phi$. In the GYRO simulations we consider only electrons and ions, assuming a trace impurity, and use $\omega$ and $\phi$ as inputs for the model represented by Equation (2). Since the main species are very weakly affected by the poloidal variation of the neoclassical potential, it does not pose a problem that GYRO assumes poloidally symmetric distribution functions. To our knowledge, presently there exists no gyrokinetic simulation code that consistently accounts for finite orbit width effects arising due to $U_{i} \sim 1$, which is also the case in GYRO; accordingly such effects are not taken into account in the gyrokinetic simulations, although $U_{i} \sim 1$ in the pedestal. The simulations use GK ions and drift kinetic electrons with $\left(m_{i} / m_{e}\right)^{1 / 2}=60$, and include electron-ion collisions. The following resolution parameters are used: 8 energies, 8 pitch angles, highest energy grid point at $v=6 v_{a}, 6$ radial grid points, 10 poloidal grid points along the orbit for circulating particles, and a time step of $\delta t=0.01 a / c_{s}$.

Figure 3a and $\mathrm{b}$ show the $k_{y} \rho_{s}$ spectra of real frequencies $\omega_{r}$ and growth rates $\gamma$ for the cases $r / a=0.975$ and $r / a=0.988$, respectively. Here, $\rho_{s}=c_{s} / \Omega_{i}$ denotes the ion sound Larmor radius. At the inner radial position for lower wave number the mode is propagating in the electron diamagnetic direction (corresponding to $\omega_{r}>0$ according to the sign convention of GYRO), then the propagation direction changes at wave numbers $k_{y} \rho_{s} \approx 0.44$, as seen in Fig. 33. We note that a mode with qualitatively similar frequency spectrum and mode structure exists at zero collision frequency for the same plasma parameters. The difference is that at $\nu_{e i}=0$, $\gamma$ is somewhat higher, $\omega_{r}$ is smaller, and the sign change in frequency appears closer to $k_{y} \rho_{s}=0.3$. At the outer radial position the mode frequency remains positive over the plotted $k_{y} \rho_{s}$ range, and the magnitude of the complex frequency is considerably higher than unity. The latter is due to the very high density gradient and a corresponding high diamagnetic frequency. We note, without showing specific GYRO results, that at $\nu_{e i}=0$ there exists a mode with qualitatively similar properties in this case as well. Although one might categorize these modes trapped electron modes based on their propagation direction, it is important to point out that the electron collision frequency is comparable to the typical bounce frequency of trapped electrons in these cases. 

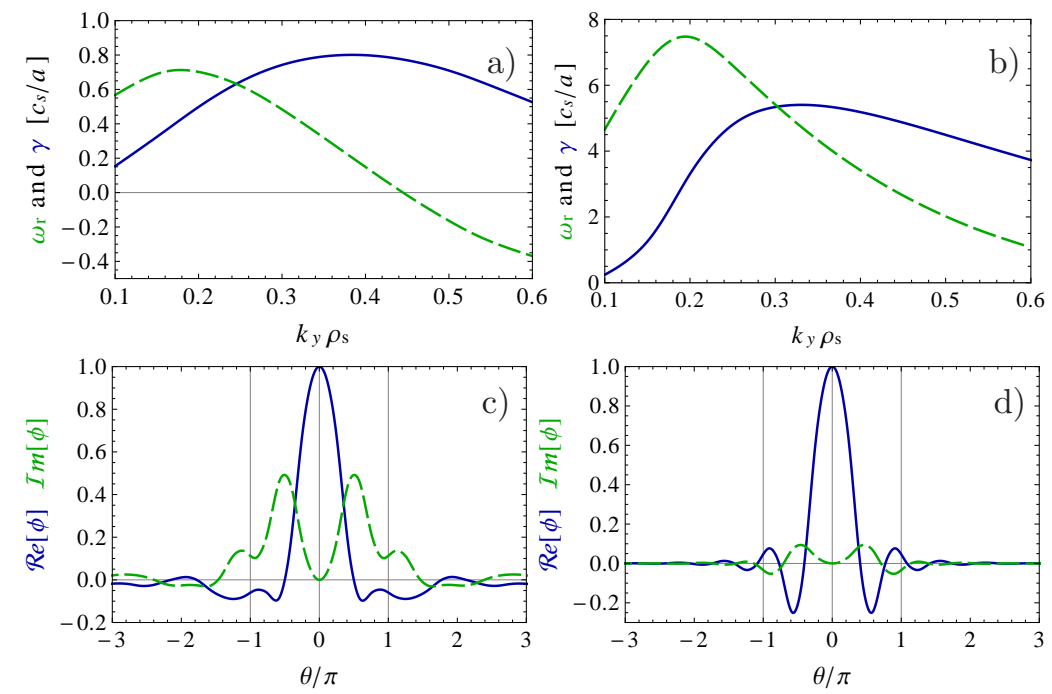

Fig. 3 a) and b) Binormal wave number spectra of $\omega_{r}$ (dashed line) and $\gamma$ (solid line) at $r / a=0.975$ and $r / a=0.988$, respectively. c) and d) Eigenfunctions $\phi$ as a function of $\theta$ corresponding to $k_{y} \rho_{s}=0.3$ of figures a) and b), respectively. Normalization: $\phi(0)=1$. Solid lines represent the real- and dashed lines represent the imaginary parts of $\phi$. Vertical grid lines mark $\theta=\{-\pi, 0, \pi\}$.

The eigenfunctions at $k_{y} \rho_{s}=0.3$ are shown in Fig. 3k and d. The modes are rather well localized in the extended poloidal angle interval $\theta \in[-\pi, \pi]$, thus we will neglect contributions outside this region for the analysis of the peaking factors.
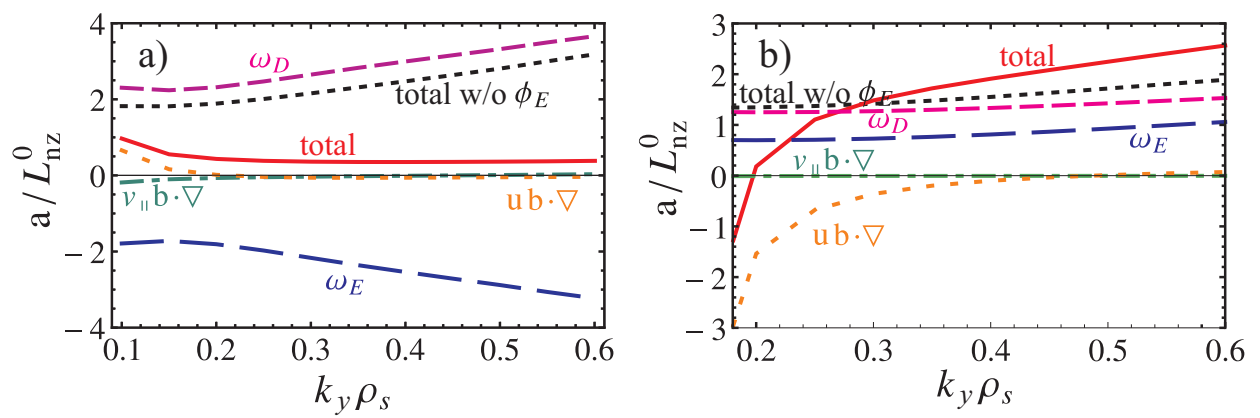

Fig. 4 Impurity peaking factors as a function of binormal wave number and contributions from the different terms of the model Eq. 2. a) is at $r / a=0.975$, b) is at $r / a=0.988$. The peaking factor in the presence of poloidal asymmetries (total) is represented by the solid line, and the contributions from the magnetic drift $\left(\omega_{D}\right)$, radial $\mathbf{E} \times \mathbf{B}$ drift $\left(\omega_{E}\right)$, parallel compressibility $\left(v_{\|} \mathbf{b} \cdot \nabla\right)$ and poloidal $\mathbf{E} \times \mathbf{B}$ drift $(u \mathbf{b} \cdot \nabla)$ are represented by the dashed, the long dashed, the dash-dotted and the long dotted lines respectively. For comparison the peaking factor for the same parameters neglecting all electric field effects (total w/o $\phi_{E}$ ) is plotted with short dotted line.

Taking the mode frequencies and eigenfunctions as input we calculate the impurity peaking factor using the model represented by Eq. (2). Note that while we take into account the shaping of the flux surface in GYRO simulations, we consider a circular cross section plasma when the peaking factors are calculated.

Figures $4 \mathrm{k}$ and $\mathrm{b}$ show the impurity peaking factors (total, solid curves) as functions of the binormal wave number, $k_{y} \rho_{s}$, for $r / a=0.975$ and $r / a=0.988$, respectively. The contributions from the magnetic drifts $\left(\omega_{D}\right.$, dashed), the radial $\mathbf{E} \times \mathbf{B}$ drift ( $\omega_{E}$, long dashed), the parallel compressibility $\left(v_{\|} \mathbf{b} \cdot \nabla\right.$, dash-dotted), and the poloidal $\mathbf{E} \times \mathbf{B}$ drift ( $u \mathbf{b} \cdot \nabla$, long dotted) are also shown in the figure. Since the parallel compressibility term is 
proportional to $1 / q^{2}$ which is typically a small number close to the LCFS, the effect of parallel compressibility is negligibly small.

On the other hand, magnetic drifts are very important, especially the radial part of the drift which is proportional to the magnetic shear that is high at the edge. The $\omega_{D}$ term is always positive, and it exhibits a weak dependence on $k_{y} \rho_{s}$ due to the weak broadening of the eigenfunction with this parameter. The magnitude of the contribution of $\omega_{E}$ to the impurity peaking is comparable to that of the magnetic drifts, as expected since $K \sim \epsilon$. In the in-out asymmetric case of $r / a=0.975$ the $\omega_{E}$ term acts to cancel the effect of the magnetic drifts, reducing the impurity peaking factor to almost zero. In the out-in asymmetric case of $r / a=0.988$ the $\omega_{E}$ term adds to the effect of the magnetic drifts leading to a substantial positive peaking factor at higher values of $k_{y} \rho_{s}$. It is interesting to note that the contribution from the magnetic drift is higher at $r / a=0.975$ than at $r / a=0.988$. The reason is that the radial part of the magnetic drift is $\propto s \theta \sin \theta$, thus it is weighted towards the inboard side. In the expression for the peaking factor this function is multiplied by the poloidally varying impurity density which, in this case, also peaks at the inboard side. This shows that the poloidal variation of $\mathcal{N}$ in combination with the magnetic drifts do not necessarily lead to a reduction of the peaking factor, especially for high magnetic shear. This observation is consistent with the trend seen in Fig. 1 of Ref. [9].

For lower wave numbers $k_{y} \rho_{s}<0.3$, where usually the nonlinear flux spectra peaks, the variation of the drift terms with $k_{y}$ is very weak. However, both the $\propto U_{i}^{2}$ and the $\propto U_{i}$ terms of (2) are inversely proportional to $k_{y}$, thus they can be non-negligible for long wavelengths. At $r / a=0.975$ (Fig. 4h) $U_{i}=0.09$, and the contribution from the $U_{i}^{2}$ term is negligibly small, the positive contribution from $u \mathbf{b} \cdot \nabla$ around $k_{y} \rho_{s}=0.1$ comes from the $U_{i}$ term and is a result of the small but finite up-down asymmetry. At $r / a=0.988$ (Fig. (4b) $U_{i}=0.68$, and although the $U_{i}^{2}$ term is non-negligible anymore it is still the $U_{i}$ term that dominates the contribution from $u \mathbf{b} \cdot \nabla$. When $\operatorname{Im}[\phi(\theta) / \phi(0)] \ll \operatorname{Re}[\phi(\theta) / \phi(0)]$, the $U_{i}$ term is approximately proportional to $\omega_{r} / \gamma$ that becomes large around $k_{y} \rho_{i}=0.1$ (see Fig. [3b). This trend, combined with the $k_{y}^{-1}$ dependence of the $U_{i}$ term, makes this term much bigger than all the other terms for small $k_{y} \rho_{s}$. Accordingly, results below $k_{y} \rho_{s}=0.18$ are not shown in Fig. 4b as the perturbative treatment of the $U_{i}$ term breaks down.

As a comparison, we also show the impurity peaking factors for the same plasma parameters, but without the effects of the radial and poloidal electric fields; see the corresponding dotted curves (total w/o $\phi_{E}$ ) in Figs. (4a-b. In general, this curve is close to, but do not exactly coincide with the magnetic drift contribution (dashed curves). Most of the difference is not due to the parallel compressibility term, but rather the fact that in the presence of $\phi_{E}$, such as for the $\omega_{D}$ curve, the weighting factor $\mathcal{N}$ has a poloidal variation. Notice, that the peaking factor without poloidal asymmetries is smaller than the magnetic drift term with poloidal asymmetries when the impurity density is peaked on the inboard side, and vice versa, again, in accordance with the poloidal variation of $\mathcal{N}$ and the $s \theta \sin \theta$ geometric factor. The general observation is that the peaking factor can be significantly modified when effects of the background electric field are taken into account; as seen comparing the solid and the dotted lines of Figs. 4 a-b.

\section{Discussion and Conclusions}

We have derived an expression for the impurity density peaking factor with corrections relevant in a steep density pedestal with subsonic ion flows. Based on realistic edge density and temperature profiles of a pedestal and a shaped magnetic geometry we calculate the electric field corresponding to the neoclassical poloidal variation of the main ion density. We use a global neoclassical solver called PERFECT that accounts for finite orbit width effects. These effects arise in a pedestal due to the sharp profile variations and strong radial electric fields. Finally, we consider the effect of the poloidal and radial electric fields on the impurity peaking due to turbulence.

We find that the poloidal location of the maximum impurity density can vary on a short radial scale in the vicinity of the pedestal. In the innermost radius of the simulation domain, there is an up-down asymmetry that transits to an in-out asymmetry, which flips to an out-in asymmetry in the strong density gradient region, then back to an in-out asymmetry again, just inside the last closed flux surface. The exact variation of the poloidal potential found here is not expected to be a universal feature, it depends on the plasma parameter profiles. However, non-negligible poloidal electric fields and their rapid variation radially may be expected in sufficiently sharp pedestals. 
We find that neoclassical asymmetries in the edge can be strong enough to significantly modify the turbulent impurity peaking, even though the profiles used here for the neoclassical calculation are not specifically chosen to maximize this effect. Due to the high safety factor and magnetic shear close to the LCFS, the effect of parallel compressibility is negligibly small compared to that of the magnetic-, and the radial $\mathbf{E} \times \mathbf{B}$ drifts. The latter can reduce or enhance the peaking factor depending on whether the poloidally varying potential is such that the impurity density peaks in the inboard or the outboard side. Due to the high magnetic shear close to the LCFS the radial part of magnetic drifts is more important than the poloidal part.

When the contribution of the $\mathbf{E} \times \mathbf{B}$ drift to the poloidal motion of the ions is non-negligible compared to that of the parallel streaming $\left(U_{i} \sim 1\right)$, as in the pedestal studied here, additional terms become important in the expression for the impurity peaking factor (2). One of them is $\propto U_{i}$ and it is finite when there is some up-down variation of the long wavelength potential $\phi_{E}$. The other one is formally similar to the parallel compressibility term, but larger by a factor $2\left(m_{z} / m_{i}\right) U_{i}^{2}$. In our case study, even though the up-down component of the asymmetry is small at the studied radii, the $\propto U_{i}$ term has a significant contribution to the impurity peaking factor, especially at small values of $k_{y} \rho_{s}$.

We note that we neglected impurity-ion friction effects in the neoclassical calculation which may also modify the poloidal variation of the impurity density. While a sharp density pedestal can be handled by the neoclassical simulation code used here, the code relies on the assumption of a slow ion temperature variation. This simplifying assumption could only be relaxed in a nonlinear, full- $f$ kinetic code.

Acknowledgements This work was funded by the European Communities under Association Contract between EURATOM and Vetenskapsrådet (VR). IP was supported by the International Postdoc grant of VR. ML was supported by the Fusion Energy Postdoctoral Research Program administered by the Oak Ridge Institute for Science and Education. Fruitful discussions with S Moradi and valuable comments from the unknown referee are gratefully acknowledged.

\section{References}

[1] M. Porkolab et al, Plasma Phys. Control. Fusion 54, 124029 (2012).

[2] C. Angioni et al, Proceedings of the $40^{\text {th }}$ EPS Conference on Plasma Physics, P4.142 (2013).

[3] P. Mantica et al, Proceedings of the $40^{\text {th }}$ EPS Conference on Plasma Physics, P4.141 (2013).

[4] T. Pütterich et al, Proceedings of the $40^{\text {th }}$ EPS Conference on Plasma Physics, I2.106 (2013).

[5] A. Kallenbach et al, Plasma Phys. Control. Fusion 47, B207 (2005).

[6] R. Neu et al, J. Nucl. Mater. 313316, 116 (2003).

[7] R. Dux et al, Plasma Phys. Controlled Fusion 45, 1815 (2003).

[8] M. Valisa et al, Nucl. Fusion 51, 033002 (2011).

[9] T. Fülöp and S. Moradi, Phys. Plasmas 18, 030703 (2011).

[10] S. Moradi, T. Fülöp, A Mollén, and I. Pusztai, Plasma Phys. Control. Fusion 53, 115008 (2011).

[11] A. Mollén, I. Pusztai, T. Fülöp, Ye. O. Kazakov, and S. Moradi Phys. Plasmas 19, 052307 (2012).

[12] A. Mollén, I. Pusztai, T. Fülöp, and S. Moradi, Phys. Plasmas 20, 032310 (2013).

[13] I. Pusztai, A. Mollén, T. Fülöp, and J. Candy, Plasma Phys. Control. Fusion 55, 074012 (2013).

[14] L. C. Ingesson, H. Chen, P. Helander, and M. J. Mantsinen, Plasma Phys. Controlled Fusion 42, 161 (2000).

[15] K. D. Marr et al, Plasma Phys. Controlled Fusion 52, 055010 (2010).

[16] R. M. Churchill et al, 54th Annual Meeting of the APS Division of Plasma Physics, BO7.008 (2012).

[17] T. Pütterich, E. Viezzer, R. Dux, R. M. McDermott, and the ASDEX Upgrade Team, Nucl. Fusion 52, 083013 (2012).

[18] M. Landreman, T. Fülöp, and D. Guszejnov, Phys. Plasmas 18, 092507 (2011).

[19] T. Fülöp and P. Helander, Phys. Plasmas 6, 3066 (1999).

[20] T. Fülöp and P. Helander, Phys. Plasmas 8, 3305 (2001).

[21] P. J. Catto, F. I. Parra, G. Kagan, J. B. Parker, I. Pusztai, and M. Landreman, Plasma Phys. Control. Fusion 53, 054004 (2011).

[22] M. Landreman and D. R. Ernst, Plasma Phys. Control. Fusion 54, 115006 (2012).

[23] R. O. Dendy, R. J. Hastie, K. G. McClements, and T. J. Martin Phys. Plasmas 2, 1623 (1995).

[24] Ye. O. Kazakov, I. Pusztai, T. Fülöp, and T. Johnson, Plasma Phys. Control. Fusion 54, 105010 (2012).

[25] R. J. Groebner and T. H. Osborne, Phys. Plasmas 5, 1800 (1998).

[26] R. J. Groebner, T. H. Osborne, A. W. Leonard, and M. E. Fenstermacher, Nucl. Fusion 49, 045013 (2009).

[27] G. Kagan and P. J. Catto, Plasma Phys. Control. Fusion 52, 055004 and 079801 (2010).

[28] I. Pusztai and P. J. Catto, Plasma Phys. Control. Fusion 52, 075016 and 119801 (2010).

[29] R. L. Miller, M. S. Chu, J. M. Greene, Y. R. Lin-Liu, and R. E. Waltz, Phys. Plasmas 5, 973 (1998).

[30] C. Angioni, F. J. Casson, C. Veth, and A. G. Peeters, Phys. Plasmas 19, 122311 (2012).

[31] J. Candy and R. E. Waltz, J. Comp. Phys 186, 545 (2003). 